\title{
Analysis of the energy spectrum of indium antimonide quantum dots with temperature changes
}

\author{
V. F. Kabanov, A. I. Mikhailov, M. V. Gavrikov \\ Saratov State University, Department of Nanoand Biomedical Technologies, \\ Astrakhanskaya, 83, Saratov, 410012, Russia \\ maks.gavrikov.96@gmail.com
}

DOI 10.17586/2220-8054-2021-12-1-113-117

\begin{abstract}
In this paper we have analyzed the broadening of the levels of the energy spectrum of indium antimonide quantum dots with a change of sample temperature. The position of the levels was determined by processing normalized differential tunneling current-voltage characteristics using the "cubic" model of a quantum dot. Comparison of the calculated values of spectrum broadening with experimental results showed qualitative and quantitative agreement between the results. It is concluded that with a decrease in the quantum dot size and, accordingly, an increase in the energy gap $\varepsilon_{c 1}-\varepsilon_{v 1}$, the broadening in percentage will decrease, which should lead to an increase in the temperature stability of the electrophysical parameters.
\end{abstract}

Keywords: quantum dots, indium antimonide, differential tunnel current-voltage characteristics, energy spectrum.

Received: 9 November 2020

Revised: 22 January 2020

\section{Introduction}

Semiconductor quantum dots (QDs) are very interesting and promising objects from both scientific and practical points of view. Currently, a fairly large number of various effective electronic and optoelectronic devices have already been created on their basis, primarily lasers and photodetectors [1]. This became possible due to their unique optical characteristics [2,3], and especially because of the narrow symmetrical fluorescence peak, which position is adjusted by changing the characteristic size, composition and form of the QDs.

Another important feature of QDs - temperature stability over the emission spectrum, in many cases observed up to room temperatures, which is associated with the three-dimensional localization of charge carriers.

One of the most popular areas of application of semiconductor QDs is creating efficient heterostructure lasers with QDs as an active medium [4,5]. This possibility is largely determined by the carrier relaxation rate. Carrier relaxation in quantum dots differs significantly from relaxation in quantum wires and quantum wells or in bulk materials. The discrete structure of the energy levels of electrons and holes in quantum dots imposes a number of restrictions on relaxation processes. Among the main factors affecting relaxation are the spectrum of electronic energy states of quantum dots, electron-hole interaction (exciton effect), the phonon states spectrum, electron-phonon interaction, the presence of excess carriers in quantum dots, as well as experimental conditions, in particular the temperature of the samples [6].

Quantum dots based on InSb are of particular interest due to the unique properties of indium antimonide: low effective masses of electrons ( $0.013 m_{0}, m_{0}$ - mass of a free electron) and, accordingly, a de Broglie wavelength up to $55 \mathrm{~nm}$, which is bigger than in other materials. This makes it possible to use this material in a variety of optoelectronic devices, sensors, IR detectors, etc. [7,8].

The use of indium antimonide quantum dots relieves, to some extent, one of the main problems of this material the significant temperature instability of the electrophysical properties associated with the small band gap of this material $(\sim 0.17 \mathrm{eV})$. With a characteristic size of InSb QDs about $10 \mathrm{~nm}$, the value of the energy gap $\varepsilon_{c 1}-\varepsilon_{v 1}$ is about $0.8 \mathrm{eV}$, which should lead to a significantly higher temperature stability of the electrophysical properties.

The purpose of this work was to study the influence of the temperature of macroscopic samples with InSb quantum dots on their electronic energy spectrum. 


\section{Sample preparation technologies and research methods}

Colloid synthesis of InSb QDs was carried out in anhydrous oleylamine using indium chloride $\mathrm{InCl}_{3}$ and antimony tris[bis(trimethylsilyl)amide] of $\mathrm{Sb}\left[\mathrm{N}\left(\mathrm{Si}-(\mathrm{Me})_{3}\right)_{2}\right]_{3}$ as precursors according to the technique $[9,10]$. An additional modification of the technique was that a mixture of acetate and indium chloride in the ratio 4:1 was used as the indium precursor. The halide in this system is necessary for the reaction, and the addition of acetate allows to minimize the aggregation processes. QD were transferred from a colloidal solution to the glass substrates with indium-tin oxide (ITO) layer by self-organization of ensembles on the surface with subsequent controlled evaporation of the solvent and control of the layer parameters. The synthesis of InSb quantum dots was carried out in the environment of oleylamine. Oleylamine ligands stabilize and passivates their surface and preventing their conglomeration by forming a shell. This shell is non-conductive and it acts as a "tunnel gap" or part of it in the system "probe - tunnel gap - quantum dot" in STM measurements.

The obtained samples were studied by scanning tunneling microscopy (STM) using a SOLVER Nano scanning probe microscope, as well as using a Zetasizer Nano ZS laser particle size analyzer. The research technique using the analysis of normalized differential tunneling CVCs is described in [11]. Additionally, in this work, measurements were carried out at sample temperatures of $23{ }^{\circ} \mathrm{C}$ and $95^{\circ} \mathrm{C}$.

In quantum dots the energy spectrum is discrete, and the distribution of the density of states depending on the energy of the charge carrier (electron) $g(\varepsilon)$ is described using the Dirac $\delta$-function and theoretically represents a set of infinitely narrow and high peaks:

$$
g(\varepsilon)=\sum_{i} \delta\left(\varepsilon-\varepsilon_{i}\right)
$$

where $\varepsilon_{i}-$ is the energy of the $i$-level of the discrete QD spectrum.

The discreteness of the energy spectrum leads to the appearance of practically atomically narrow lines in the luminescence (or absorption) spectrum of a QDs. Direct observation of narrow lines for an ensemble of QDs is impossible due to a sufficiently large scatter of the QDs parameters, which leads to a noticeable inhomogeneous line broadening.

When using the model of a cubic QD with edge a, the value of the energy of the discrete level can be determined as [12]:

$$
\varepsilon_{i}=\frac{(\pi \hbar)^{2}}{2 m^{*}} \cdot \frac{1}{a^{2}} \cdot\left(l^{2}+m^{2}+n^{2}\right),
$$

where $l, m, n=1,2,3 \ldots ; m^{*}$ is effective mass of electron in a bulk material; $a$ is the characteristic size of QD.

The carrier scattering leads to broadening of energy levels and limiting the height of the peaks: $\Delta \varepsilon_{i} \sim \hbar \tau$, where $\tau$ is the lifetime of electron in quasistationary states of QDs [13].

Such broadening in a real QDs occurs because of the limited lifetime of charge carriers due to spontaneous emission, interaction with phonons, temperature fluctuations, and some other processes.

The magnitude of broadening $\Delta \varepsilon_{i}$ is related to the value $\varepsilon_{i}$. Both of these parameters affect the form and character of the $g(\varepsilon)$ dependence.

The nature and magnitude of the broadening $\Delta \varepsilon_{i}$, in accordance with the concepts presented by the authors of [13], in the case of simple energy bands with a quadratic dispersion law, taking into account the corresponding simplifications, can be represented by the equation:

$$
\Delta \varepsilon_{i} \approx 16 \frac{\varepsilon^{1 / 2}}{\varepsilon_{i}}\left(\varepsilon_{i}-\varepsilon\right)^{3 / 2} \cdot e^{-2 \Delta r\left(2 m^{*}\left(\varepsilon_{i}-\varepsilon\right)^{1 / 2} / \hbar\right)},
$$

where $\Delta r$ is QD shell thickness.

The value of $\varepsilon_{i}$ is largely determined by the characteristic size of the QDs, the composition and form of the QDs and, thus, is largely determined by the QDs producing technology.

\section{Results and discussion}

We studied samples of indium antimonide quantum dots with a characteristic size $8-10 \mathrm{~nm}$ (Fig. 1). Magnitude estimate of the first energy spectrum level $\varepsilon_{1}$ in accordance with (2) was in the range from 0.8 to $1.5 \mathrm{eV}$.

Localized energy levels $\varepsilon_{i}$ associated with the properties of a quantum-size object appear in normalized differential tunneling CVCs as individual peaks. To analyze the experimental tunneling $\mathrm{I}-\mathrm{V}$ characteristics, similarly to the approaches described in $[14,15]$, we used the dependence of $(d I / d V) /(I / V)$ on the voltage $V$, since the method of normalized differential CVCs is more informative in experimental data processing. Typical result for investigated samples are shown in Fig. 2. 


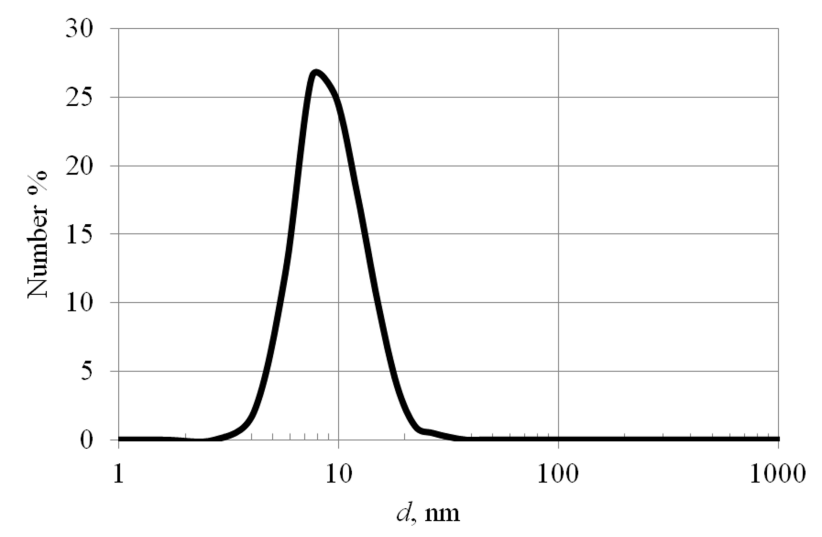

FIG. 1. Determination of the characteristic size of InSb QDs using a laser particle size analyzer

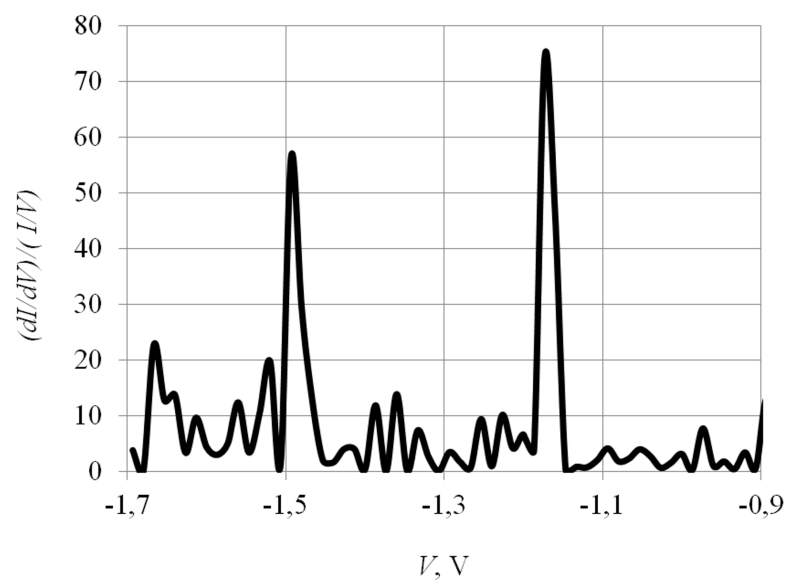

FIG. 2. Typical normalized differential tunneling CVC of InSb QDs

A change in the energy of the system, for example, upon heating the samples, should affect the broadening $\Delta \varepsilon_{i}$ in accordance with (3) and lead to changes in the energy spectrum of QDs. Typical experimental results of normalized differential CVCs are shown in Fig. 3.

An analysis of the experimental results showed that the position of the peak corresponding to the local energy levels of QDs shifts by $3-5 \%(0.03-0.04 \mathrm{~V})$. This is within the measurement accuracy (about $2 k T)$, but nevertheless the statistically reproducible data allow us to conclude that the results are not a measurement error.

In accordance with (3), Fig. 4 shows the calculated values of the broadening of the first energy level of InSb QDs upon heating to $95{ }^{\circ} \mathrm{C}$. In the calculations, we chose the energy intervals $2 k T$ relative to $\varepsilon_{1}$. Estimation shows that in the considered energy range corresponding to the characteristic QDs sizes, the broadening of the first level is in the range from 2.5 to $4.0 \%$, depending on the level energy (the QDs shell thickness was chosen as $2 \mathrm{~nm}$ ).

Comparison with the experimental results (Fig. 3) showed a fairly good agreement between the calculated and experimentally determined broadening of the first energy level of InSb QDs.

It should be noted that in the analysis of the broadening of the QDs energy levels, other factors were not taken into account, for example, the spectrum of phonon states, electron-phonon interaction and ect.

Obviously, a change in the value of $\Delta \varepsilon_{1}$ will affect the width and energy position of the fluorescence (or absorption) peak and, accordingly, the characteristics of devices with quantum dots in their structure. In this regard, the analysis of this issue is absolutely necessary. However, as can be seen from the calculation and experiment, the broadening of the first energy level of the QDs for the considered characteristic sizes and the selected temperature range does not exceed $2 k T$ (which is within the measurement accuracy). Therefore, an increase in temperature in considered range has practically no effect on the energy characteristics of the QD. With a decrease in the QD size and, accordingly, an increase in the energy gap $\varepsilon_{c 1}-\varepsilon_{v 1}$, the broadening in percentage will decrease (Fig. 4), which should further increase the temperature stability of the parameters. 


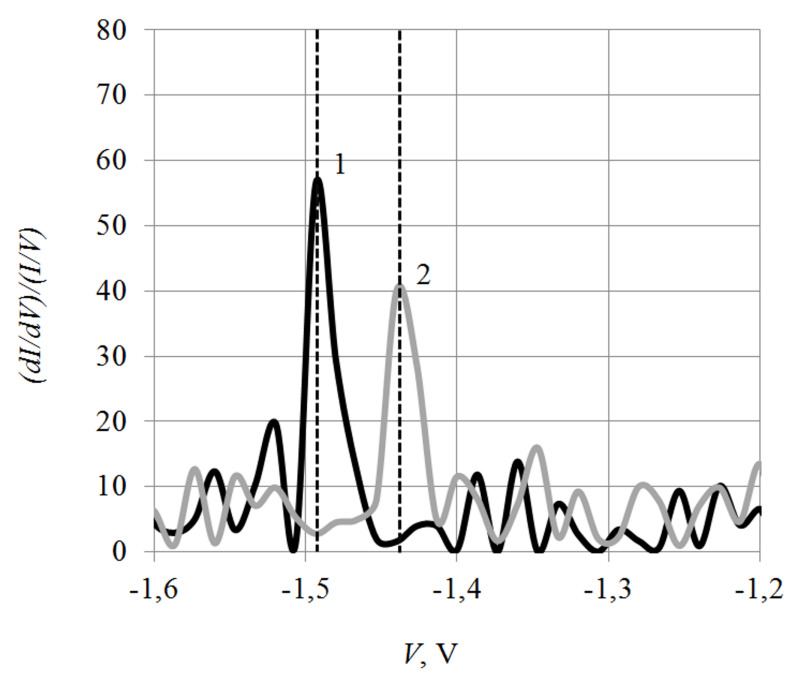

FIG. 3. Typical normalized differential tunneling CVCs of InSb QDs at two samples temperatures: $1-23{ }^{\circ} \mathrm{C}, 2-95{ }^{\circ} \mathrm{C}$

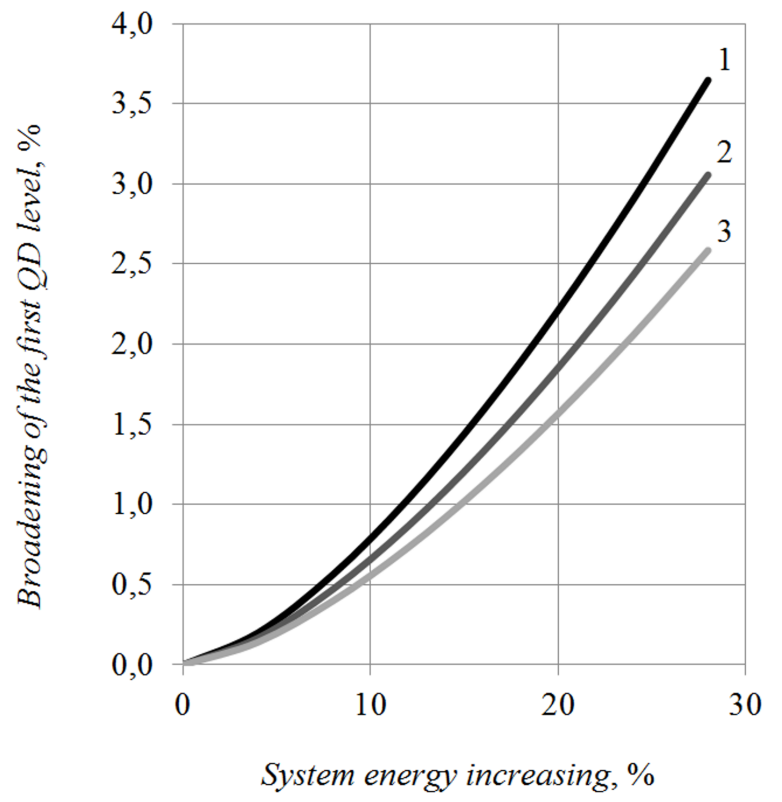

FIG. 4. The calculated values of the broadening of the first level of InSb QDs at various specified values of $\varepsilon_{1}: 1-0.7 \mathrm{eV}, 2-1.0 \mathrm{eV}, 3-1.4 \mathrm{eV}$

\section{Conclusion}

Thus, we analyzed the effect of temperature change on the energy spectrum of InSb quantum dots regarding broadening of the energy spectrum levels. The position of the levels was determined by processing normalized differential tunneling CVCs using the "cubic" QD model. Comparison of the calculated values of spectrum broadening with experimental results showed qualitative and quantitative agreement.

It was concluded that the broadening of the first QDs energy level, which is the most important in practice applications, for the considered characteristic sizes does not exceed $2 k T$ (which is within the measurement accuracy), i.e. a change in temperature does not significantly affect the change in the energy characteristics of QDs in the considered temperature range. With a decrease of the QDs size and, accordingly, an increase in the energy gap $\varepsilon_{c 1}-\varepsilon_{v 1}$, the broadening of the first energy level of the QDs in percentage will decrease, which should lead to an increase in the temperature stability of the parameters of electronic and optoelectronic devices using InSb QDs. 
The obtained results can be used to study the effect of temperature operations regimes on the optical and electronic characteristics of devices with semiconductor quantum dots.

\section{Acknowledgements}

This work was supported by grants from the Russian Foundation for Basic Research Projects No. 19-07-00087 and No. 19-07-00086.

\section{References}

[1] 1Fedorov A.V. Optics of nanostructures. Nedra, St.-Petersburg, 2005, $326 \mathrm{p}$

[2] Oleinikov V.A., Sukhanova A.V., Nabiev I.R. Fluorescent semiconductor nanocrystals in biology and medicine. Russian nanotechnology, 2007, 2, P. 160-173.

[3] Sukhanova A.V., Venteo L., et al. Highly Stable Fluorescent Nanocrystals as a Novel Class of Labels for Immunohistochemical Analysis of Paraffin-Embedded Tissue Sections. Lab Invest, 2002, 82, P. 1259-1261.

[4] Ledentsov N.N., Grundmann M., et al. Quantum-dot heterostructure lasers. Journal of Selected Topics in Quantum Electronics, 2000, 6 (3), P. 439-451.

[5] Bimberg D. Quantum dots for lasers, amplifiers and computing. Journal of Physics D Applied Physics, 2005,38 (13), P. $2055-2058$.

[6] Ignatiev I.V., Kozin I.E. Carrier dynamics in semiconductor quantum dots. St.-Petersburg State university, St.-Petersburg, 2005,126 p.

[7] Reiss P., Carriere M., et al. Synthesis of Semiconductor Nanocrystals, Focusing on Nontoxic and Earth-Abundant Materials. Chem. Rev., 2016, 116 (18), P. 10731-10819.

[8] Yoffe A.D. Semiconductor quantum dots and related systems: Electronic, optical, luminescence and related properties of low dimensional systems. Advances in Physics, 2010, 50 (1), P. 1-208.

[9] Brichkin S.B., Razumov V.F. Colloidal quantum dots: synthesis, properties and applications. Russ. Chem. Rev., 2016,85 (12), P. 1297-1312.

[10] Liu W., Chang A.Y., Schaller R.D., Talapin D.V. Colloidal InSb Nanocrystals. J. Am. Chem. Soc., 2012, 134, P. 20258-20261.

[11] Mikhailov A.I., Kabanov V.F., Zhukov N.D., Glukhovskoy E.G. Features of the energy spectrum of quantum dots indium antimonide. Nanosystems: Physics, Chemistry, Mathematics, 2017, 8 (5), P. 596-599.

[12] Dragunov V.P., Neizwestny I.G., Gridchin V.A. Fundamentals of nanoelectronics. Fizmatkniga, Moscow, 2006,496 p.

[13] Zegrya G.G., Samosvat D.M. Energy spectrum and lifetime of charge carriers in open quantum dots in an electric field. Journal of Experimental and Theoretical Physics, 2009, 135 (6), P. 1043-1055.

[14] Mikhailov A.I., Kabanov V.F., Gorbachev I.A., Glukhovsky E.G. Study of the properties of $\mathrm{A}^{I I} \mathrm{~B}^{V I}$ and $\mathrm{A}^{I I I} \mathrm{~B}^{V}$ semiconductor quantum dots. Semiconductors, 2018, 52 (6), P. 603-607.

[15] Mikhailov A.I., Kabanov V.F., et al. Methodology of analyzing the CdSe semiconductor quantum dots parameters. Nanosystems: Physics, Chemistry, Mathematics, 2018, 9, P. 464-467. 